Part of Journal of Research of the National Bureau of Standards, Volume 27, October 1941

\title{
LOCATING THE PRINCIPAL POINT OF PRECISION AIRPLANE MAPPING CAMERAS
}

\author{
By Francis E. Washer
}

\section{ABSTRACT}

The location of the principal point with respect to the intersection of lines joining opposite pairs of collimation index markers was determined for numerous precision airplane mapping cameras. Weak prism effect, present in most camera lenses, was measured with the Bureau's precision lens-testing camera and its influence on the location of the principal point considered. It was found that these cameras required adjustments ranging from 2 to 25 times the allowable tolerance of $\pm 0.03 \mathrm{~mm}$ in the location of the principal point.

\section{CONTENTS}

I. Introduction

II. Method

III. Interpretation of negatives

1. Perpendicularity of collimation markers

2. Locating center of collimation with respect to center cross _... _ 408

3. Distance of center cross from principal point _... _...

4. Locating principal point with respect to center of collimation _. _ 411

5. Adjustment of airplane camera _...................... 412

\section{INTRODUCTION}

Rapid advances in the use of aerial photography for map making have been accompanied by steady improvements in the airplane camera as a precision instrument. As a result of these improvements the increased accuracy obtainable on projects calling for planimetric mapping has made it feasible to use the same negatives for the preparation of topographic maps of the same area, thus eliminating the necessity of rephotographing the region.

The need for topographic maps is evidenced by the fact that the United States is the least thoroughly mapped of any of the great industrial nations. ${ }^{1}$ Such maps are of prime importance in the laying out of new road systems and in the location of proper dam sites for drainage control or power developments. With these aims in mind, various governmental agencies have interested themselves in the problems of making the negatives obtained on one project more useful for further studies of the same region.

It was soon found that negatives from different sources did not usually lend themselves readily to stereo-photogrammetric plotting of topography. Some of these difficulties were quickly traced to large values of the distortion and low resolving power, but after these factors \footnotetext{
1 C. A. Hart, Air Photography Applied to Surveying, p. 2 (Longmans, Green \& Co., New York, N. Y.,
1940).
} 
had been minimized by the establishment of minimum performance standards for the lenses used in aerial cameras, ${ }^{2}$ some difficulties still remained that were traceable to the airplane mapping camera.

Chief among these difficulties were the inadequate and nonuniform methods of showing the location of the principal point of the camera on the negative. This point is the intersection of a perpendicular dropped from the rear nodal point of the lens with the focal plane, and the importance of its accurate location has been shown in a paper by I. C. Gardner. ${ }^{3}$ To insure more reliable information on the location of the principal point on each negative, new rigid requirements ${ }^{4}$ for airplane cameras were established giving detailed instructions regarding the manner of recording index marks on each negative at the moment of exposure. Such index marks are to have a definite relation to the principal point of the camera and also to appear at the same place on all negatives from every camera being used on governmental projects.

These index marks on the negative are made by devices called the collimation index markers, set in the center of each side of the rear opening of a precision camera. They are so arranged that when each successive exposure is made, the image of each marker will be registered and show on the negative as a fine radial line. The detail requirements are that, for a camera using 9- by 9 -inch or 7 - by 9-inch films or plates and having a lens of approximately 8 inches focal length, the lines joining opposite members of the two pairs of index markers shall intersect at an angle of $90^{\circ} \pm 1$ minute ${ }^{5}$ and shall indicate the location of the principal point with a probable error not exceeding $\pm 0.03 \mathrm{~mm}$.

Since it has been a requirement for several years that all lenses and cameras used on many Government mapping projects be tested by the National Bureau of Standards, it was necessary that methods be devised for determining the location of the principal point of an airplane mapping camera. This has been done, and this paper presents a description of the method as well as a survey of the results of measurements made on a number of airplane mapping cameras.

\section{METHOD}

Analysis of the problems involved in the certification of a precision airplane camera indicated that modification of the existing precision lens-testing camera offered the best immediate solution. This apparatus has been previously described in a paper by Gardner and Case ${ }^{6}$ and no detailed description will be given here, except to mention that it has been enlarged and now has 10, instead of 7 , collimators spaced at $5^{\circ}$ intervals. For the calibration of precision airplane cameras, only nine of these collimators are used. The optical bench that carries the apparatus for the testing of photographic objectives is lifted from its mounting, and a telescope is mounted on the rear portion of the frame thus vacated. This telescope is adjusted to parallelism with the central one of the nine collimators, and centered upon the cross that serves as the collimator target.

\footnotetext{
2 Standard specifications (2d ed.) published by American Society of Photogrammetry on July 22, 1936.

3 J. Research NBS 22, 209 (1939) RP1177.

4 United States Department of Agriculture Specification No. A-APC-1102 (as approved March 1940).

5 This requirement specifying the angular positioning of the collimation index markers insures their registration in the same place on negatives from different cameras and makes possible the use of such negatives without the necessity of readjustment of the plotting machine.

6 J. Research NBS 18, 449 (1937) RP984.
} 


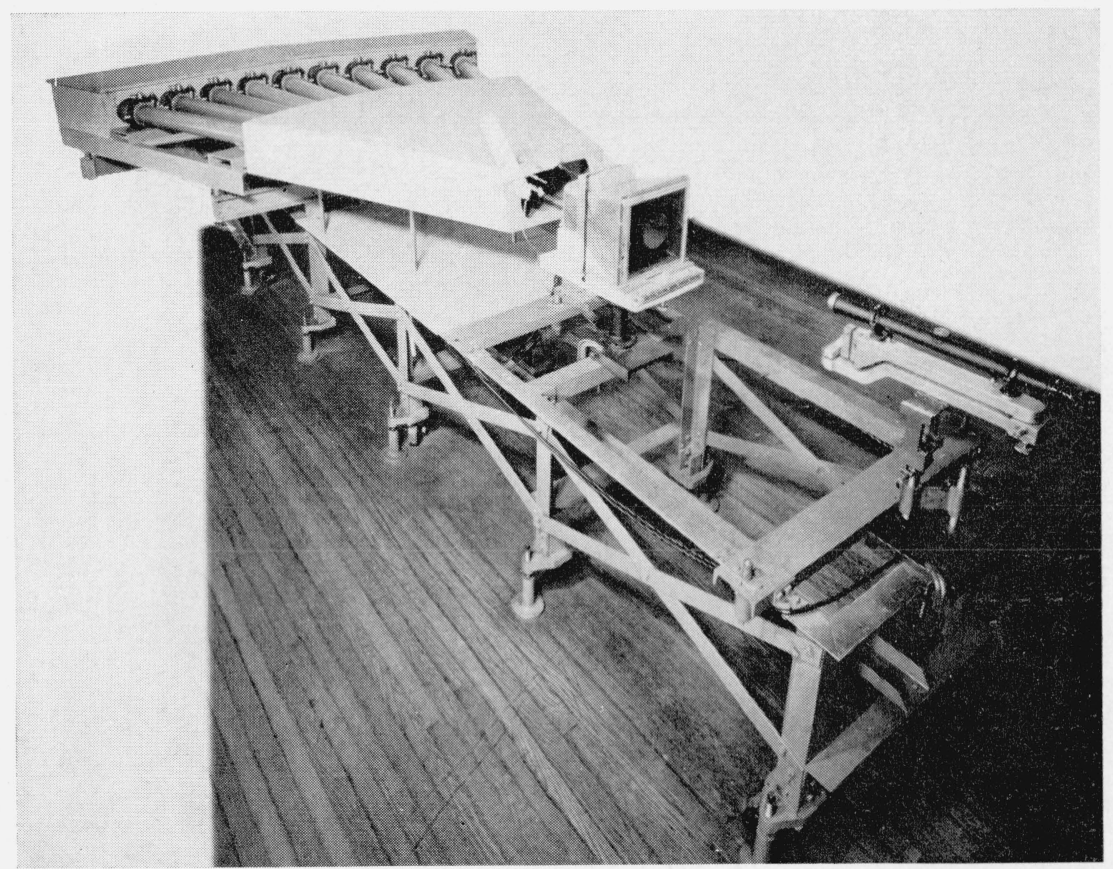

FIGURE 1.-Precision lens-testing camera with an airplane mapping camera mounted in position for test. 
The airplane camera is rigidly mounted on the platform of a stand so located that the camera lens bears the same relation to the collimators that normally prevails for a lens when tested separately. The arrangement is shown in figure 1. The stand has adjustments that permit vertical and lateral translations and rotation of the platform carrying the camera about the vertical and the transverse horizontal axes. Adjustments are made until the camera is satisfactorily centered with respect to the collimator system and is pointed at the center collimator. A silvered flat glass plate is placed on the camera back so that it is pressed against the square flat metal plate surrounding the camera opening; the surface of this metal plate determines the focal plane of the camera. The telescope, the adjustment of which is left unchanged throughout the process, is now used as an autocollimator, and the focal plane of the camera is made perpendicular to the axis of the autocollimating telescope by small rotations of the camera platform. The rotation is so constrained that there is little likelihood of drift after the desired position is reached. Since the axis of the autocollimating telescope was initially set parallel to the axis of the center collimator, the focal plane of the camera is also perpendicular to the axis of the center collimator. Under these conditions, the cross that serves as a target for the center collimator will be imaged in the focal plane and indicate the point where parallel light directed perpendicular to the focal plane is imaged. This image, or the point determined by the image, will be referred to as the center cross. The targets of the remaining eight collimators are also imaged in the focal plane of the camera, and indicate the positions where parallel rays of light at $5^{\circ}$, $10^{\circ}, 15^{\circ}$, and $20^{\circ}$ on each side of the central ray are imaged.

When the camera has been properly alined, a photographic plate is substituted for the silvered glass plate in such a manner that its emulsion surface lies in the focal plane. On exposure, the images from the nine collimators are registered; simultaneously the collimation index markers are registered by means of four small flashlight bulbs arranged in front of the camera. To reduce error, four negatives are made, the camera being rotated $90^{\circ}$ about its axis of symmetry between exposures.

\section{INTERPRETATION OF NEGATIVES}

\section{PERPENDICULARITY OF COLLIMATION MARKERS}

The first determination made from the negatives is that of the angle between the lines joining opposite collimation markers. For this purpose a piece of plate glass is used with four short radial diamond lines $90^{\circ}$ apart cut in the surface near the end of a 4.5 -inch radius. The error in the positioning of these marks does not exceed \pm 5 seconds. This plate is placed face down upon the emulsion side of the negatives in such manner that the radial diamond lines are nearly in coincidence with the index lines of the collimation markers. The plate and negative are then fastened firmly together and measurements made of the separation of each of the diamond lines from the corresponding index lines. Interpretation of the data thus gained yields the amount of departure from $90^{\circ}$ of the angle between lines joining opposite collimation markers. If the departure does not exceed \pm 1 minute, the adjustment is adjudged satisfactory. It is noteworthy that over 90 percent of the precision cameras thus far measured have passed this requirement satisfactorily. 


\section{LOCATING CENTER OF COLLIMATION WITH RESPECT TO CENTER CROSS}

The first step in the location of the principal point is the location of the position of the center cross with respect to the point of intersection of lines joining opposite collimation index marks. This point is called the center of collimation. Considering the lines joining opposite pairs of collimation index markers as the axes of a rectangular coordinate system with the center of collimation as origin, as is indicated in figure 2 , the coordinates of the center cross are obtained from the negative. These measurements are made with a micrometer micro-

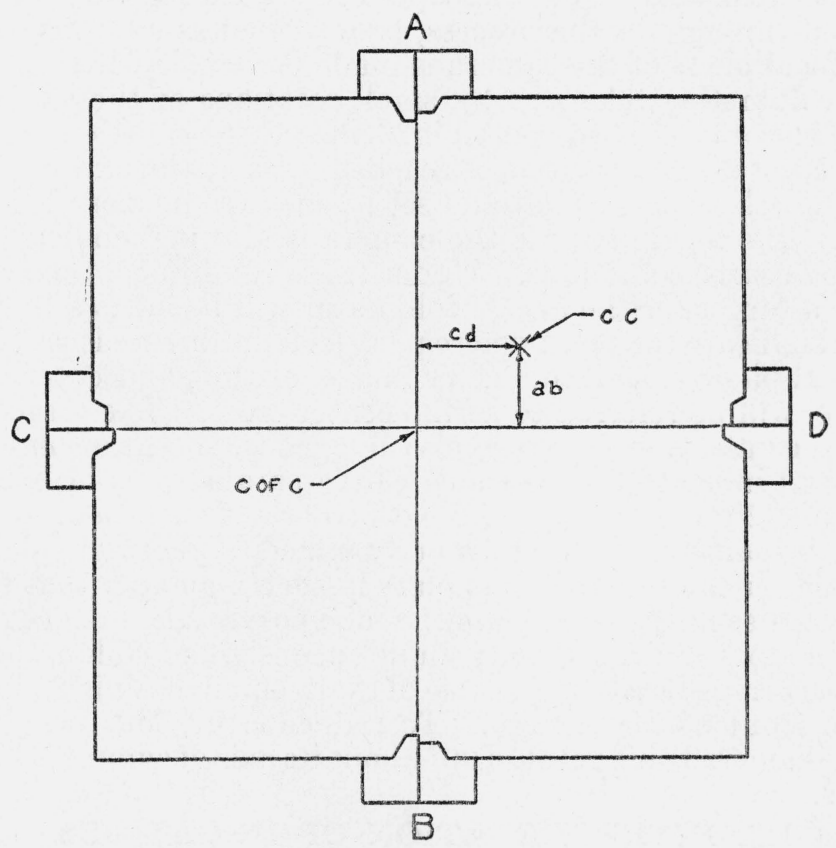

FIGURE 2.-Schematic representation of a negative made with an airplane mapping camera.

$A, B, C$, and $D$ are the collimation index marks, $X$ shows the position of the center cross $(C C)$. The intersection of lines joining opposite pairs of collimation marks is called the center of collimation $(C$ of $C$ ).

scope used, in conjunction with a slide of an optical bench, in a manner that permits measurement in a direction at right angles to the direction of movement of the slide carrying the negative. For convenience, the coordinate parallel to the line of flight is designated $a b$, and the one at right angles thereto $c d$. Measurements are made on each of the four negatives, and the final values of the coordinates are given by the average of the four determinations. Table 1 shows the magnitudes of these coordinates for a representative group of cameras and also the actual separation of the center of collimation and the center cross. The approximate probable error in the determination of each coordinate of the center cross with respect to the coordinate frame of the collimation index markers is $\pm 0.008 \mathrm{~mm}$. The approximate probable 
error in the determination of the distance between the center cross and the center of collimation is $\pm 0.011 \mathrm{~mm}$.

TABLE 1.-Distance of center cross from center of collimation

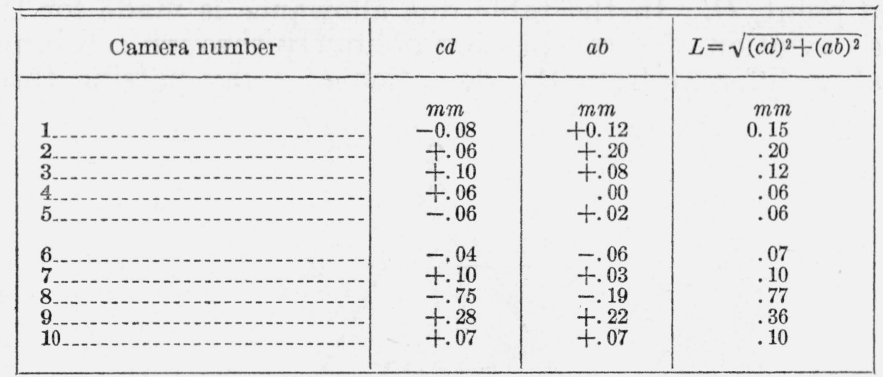

\section{DISTANCE OF CENTER CROSS FROM PRINCIPAL POINT}

The second step in the location of the principal point is to determine its position with respect to the center cross. For an ideal lens the principal point and the center cross coincide, since the principal point of a camera is defined as that point where a perpendicular dropped from the rear nodal point of the lens intersects the focal plane. In practice, the optic axis of a lens system is seldom an unbroken straight line. An axial ray may actually bend at each surface to form a broken line, and the ray that finally emerges may not be parallel to the incident ray. This effect is presumably produced by slight decentrations of the components of the lens system, and it can be approximated by treating the lens system as an ideal lens plus a weak prism. ${ }^{7}$ Since this prism effect occurs in varying amounts in most real lenses, the center cross will be shifted slightly from the principal point when the negatives are made under the conditions described in section II.

Considering the camera lens as an ideal lens plus a weak prism, it is apparent that the images from all nine collimators are shifted from the positions they normally would have occupied in a direction determined by the orientation of the effective weak prism. The deviation of a given ray is a function of the prism angle and the angle of separation from the central ray. In figure 3 , let $C$ represent the center of an ideal lens, and $C X_{1}$ and $C X_{2}$ rays making equal angles, $\beta$, with the central ray, $C O$. Let $O$ be the principal point of the camera and $C O$ the principal distance, or focal length, $f$. If a weak prism of angle $\alpha$ be interposed between the lens and the focal plane, the points of intersection of rays $C X_{2}, C O$, and $C X_{1}$ are shifted to $X_{2}^{\prime}, O^{\prime}$, and $X_{1}^{\prime}$, the direction of shift being toward the base of the prism. The angle of the shift for each ray is given by $\epsilon_{2}, \epsilon_{0}$, and $\epsilon_{1}$. From the diagram

and

$$
\begin{aligned}
& O^{\prime} X_{2}^{\prime}=f\left[\tan \left(\beta+\epsilon_{2}\right)-\tan \epsilon_{0}\right]=f \lambda, \\
& O^{\prime} X_{1}^{\prime}=f\left[\tan \left(\beta-\epsilon_{1}\right)+\tan \epsilon_{0}\right]=f \xi,
\end{aligned}
$$

$$
O^{\prime} X_{2}^{\prime}-O^{\prime} X_{1}^{\prime}=f(\lambda-\xi) \text {. }
$$

\footnotetext{
'A. H. Bennett, J. Opt. Soc. Am. and Rev. Sci. Instr. 14, 235 (1927).
} 
Table 2 shows the values of $f(\lambda-\xi)$ for a $210.00-\mathrm{mm}$ lens with $\beta=20^{\circ}$ for several values of the prism angle $\alpha$. Values of $\epsilon_{2}, \epsilon_{0}, \epsilon_{1}$, from which the values of $f(\lambda-\xi)$ are computed are also shown. The column $f \tan \epsilon_{0}$ shows the actual shift of $O^{\prime}$, the center cross from the principal point, $O$. In the table due allowance is made for the fact that the deviation of a ray passing obliquely through a thin prism is appreciably different from the deviation of a ray passing through a



FIGURE 3.-Efject of interposing a prism between lens and image plane.

The solid lines show rays proceeding from $C$, the rear nodal point of the lens, to the image plane. The dashed lines show how these rays are deviated by a thin prism of angle $\alpha$.

thin prism in the position of minimum deviation. This is evidenced by the differences in the values of $\epsilon_{1}, \epsilon_{2}$, and $\epsilon_{0}$.

TABLE 2.-Effects a of a weak prism combined with an ideal lens

\begin{tabular}{|c|r|r|r|r|c|}
\hline$\alpha$ & $\epsilon_{1}$ & $\epsilon_{0}$ & $\epsilon_{2}$ & $f(\lambda-\xi)$ & $f \tan \epsilon_{0}$ \\
\cline { 2 - 5 } \cline { 5 - 6 } Degrees & Degrees & Degrees & Degrees & $m m$ & $m m$ \\
0.01250 & 0.00693 & 0.00625 & 0.00693 & 0.0117 & 0.0229 \\
.02500 & .01385 & .01250 & .01385 & .0233 & .0458 \\
.05000 & .02771 & .02500 & .02772 & .0468 & .0916 \\
.10000 & .05538 & .05000 & .05546 & .0935 & .1833 \\
\hline
\end{tabular}

- Computed values of the angular deviation of the axial ray and of rays making angle $\beta=20^{\circ}$, with the axial ray for the combination of an ideal $210 \mathrm{~mm}$ lens and a weak prism of index 1.5 and angle $\alpha$. The values of $f(\lambda-\xi)$ are given together with the computed value of the shift, $f \tan \epsilon_{0}$, of the center cross with respect to the principal point.

A similar table has been prepared for $\beta=15^{\circ}$ and is used in conjunction with table 2 in determining the location of the center cross with respect to the principal point. In actual practice, the quantities $\mathrm{O}^{\prime} \mathrm{X}_{2}{ }^{\prime}$ and $O^{\prime} X_{1}{ }^{\prime}$ are measured from the negative and their difference forms a value of $f(\lambda-\xi)$ with which the shift of the center cross is obtained from the tables. 
To avoid the tedious process of setting the collimators at precisely equal angles, it has proved more convenient in the evaluation of $f(\lambda-\xi)$ to combine the measurements from two negatives that were made with the airplane camera in positions $180^{\circ}$ apart as measured around the axis of symmetry of the airplane camera. This procedure reduces the amount of computation and eliminates the necessity of compensating for the distortion of the lens, which is presumed to be symmetrical about the lens axis. The direction of shift of the center cross from the principal point is given by the algebraic sign of $f(\lambda-\xi)$.

The shift of the center cross on account of prism may be in any direction in the focal plane. Accordingly the coordinates $a b$ and $c d$ of the center cross with respect to the principal point are obtained by using two pairs of negatives, each pair of which gives one of the coordinates. The magnitudes of these coordinates, as well as the total shift of the principal point for a representative group of cameras, are shown in table 3 . The approximate probable error in the determination of the distance between the principal point and the center cross does not exceed $\pm 0.005 \mathrm{~mm}$. As a matter of interest the approximate values of effective prism angle are also shown.

TABLE 3.-Distance of center cross from principal point

\begin{tabular}{|c|c|c|c|c|c|}
\hline Camera number & $c d$ & $a b$ & $\begin{array}{l}\sqrt{(c d)^{2}+(a b)^{2}} \\
=f \tan \epsilon_{0}\end{array}$ & $f$ & $\alpha$ \\
\hline $\begin{array}{l}1 \\
2 \\
2 \\
3 \\
4 \\
5 \\
6 \\
7 \\
8 \\
9 \\
9\end{array} 10$ & $\begin{array}{l}m m \\
-0.02 \\
-.04 \\
-.05 \\
-.02 \\
+.06 \\
+.08 \\
+.01 \\
-.04 \\
+.16 \\
+.03\end{array}$ & $\begin{array}{l}m m \\
+0.04 \\
+.03 \\
-.03 \\
-.02 \\
+.07 \\
-.03 \\
.00 \\
+.01 \\
+.02 \\
+.01\end{array}$ & $\begin{array}{r}m m \\
0.04 \\
.05 \\
.05 \\
.03 \\
.09 \\
.09 \\
.01 \\
.04 \\
.17 \\
.03\end{array}$ & $\begin{array}{l}m m \\
209.78 \\
209.67 \\
209.67 \\
210.22 \\
210.21 \\
209.50 \\
209.65 \\
209.87 \\
210.93 \\
255.59\end{array}$ & $\begin{array}{c}\text { Minutes } \\
1.4 \\
1.6 \\
1.8 \\
1.0 \\
3.0 \\
2.9 \\
0.4 \\
1.3 \\
5.4 \\
0.8\end{array}$ \\
\hline
\end{tabular}

\section{LOCATING PRINCIPAL POINT WITH RESPECT TO CENTER OF COLLIMATION}

In section 3 , the method of locating the center cross with respect to the center of collimation is given; in section 4, the method of locating the principal point with respect to the center cross is given. Combining the results of these two processes with due regard to the geometry of the configuration, we obtain the location of the principal point with respect to the center of collimation. Values of the separation distance for a representative group of cameras are shown in table 4. It is apparent that, for the majority of cameras as submitted for certification, the measured separation of principal point and center of collimation is considerably in excess of the tolerance of $\pm 0.03 \mathrm{~mm}$. 
TABLE 4-Distance of principal point from center of collimation before adjustment of airplane camera

\begin{tabular}{|c|c|c|c|}
\hline Camera number & $c d$ & $a b$ & $\sqrt{(c d)^{2}+(a b)^{2}}$ \\
\hline $\begin{array}{l}1 \\
2 \\
3 \\
4 \\
4 \\
5\end{array}$ & $\begin{array}{c}m m \\
-0.06 \\
+.10 \\
+.14 \\
+.08 \\
-.11\end{array}$ & $\begin{array}{c}m m \\
+0.08 \\
+.17 \\
+.10 \\
+.02 \\
-.05\end{array}$ & $\begin{array}{c}m m \\
0.10 \\
.19 \\
.18 \\
.09 \\
.12\end{array}$ \\
\hline $\begin{array}{l}6 \\
7 \\
8 \\
9 \\
9\end{array}$ & $\begin{array}{l}-.12 \\
+.09 \\
-.71 \\
+.12 \\
+.04\end{array}$ & $\begin{array}{l}-.02 \\
+.03 \\
-.20 \\
+.24 \\
+.06\end{array}$ & $\begin{array}{l}.12 \\
.09 \\
.74 \\
.27 \\
.07\end{array}$ \\
\hline
\end{tabular}

\section{ADJUSTMENT OF AIRPLANE CAMERA}

Each of the cameras submitted for examination and adjustment is provided with devices that permit transverse movement of the lens with respect to the metal plate carrying the index markers. On the basis of measurements of the type described, the principal point and the center of collimation are adjusted to coincidence by appropriate movement of the lens with respect to the metal plate bearing the index markers. After adjustment, the movable part is so doweled to the camera body that this adjustment will be preserved. On account of the difficult nature of this adjustment and the possibility of slight shifts during the doweling, the location of the principal point, with respect to the center of collimation, is also measured after the doweling, and if the separation does not exceed $\pm 0.03 \mathrm{~mm}$, the adjustment is considered satisfactory.

This method has been used on a total of more than 40 precision airplane mapping cameras that have been submitted for use on projects of the Department of Agriculture. In practically all instances, it was necessary to make adjustments, as the departure of the principal point from the center of collimation ranged in the various cameras from 2 to 25 times the allowable tolerance of $\pm 0.03 \mathrm{~mm}$, and the average separation was $0.30 \mathrm{~mm}$, or 10 times the tolerance value.

Washington, August 23, 1941. 


\section{MATHEMATICAL TABLES}

Attention is invited to a series of publications which is being prepared by the Project for the Computation of Mathematical Tables conducted by the Federal Works Agency, Work Projects Administration for the City of New York under the sponsorship of the National Bureau of Standards.

To date, six tables have been made available through the National Bureau of Standards. These are listed below:

MT1. Table of the First Ten Powers of the Integrrs From 1 to 1000:

(1938) VIII + 80 pages; heavy paper cover. 50 cents.

MT2. Tables of the Exponential Function $e^{x}$ :

The ranges and intervals of the argument and the number of decimal places in the entries are given below:

$\begin{array}{ccc}\text { Range of } x & \text { Interval of } x & \text { Decimals given } \\ -2.5000 \text { to } 1.0000 & 0.0001 & 18 \\ 1.0000 \text { to } 2.5000 & .0001 & 15 \\ 2.500 \text { to } 5.000 & .001 & 15 \\ 5.00 \text { to } 10.0 & .01 & 12\end{array}$

(1939) XV+535 pages; bound in buckram, $\$ 2.00$.

MT3. Tables of Circular and Hyprrbolic Sines and Cosines for Radian Arguments:

Contains 9 decimal place values of $\sin x, \cos x, \sinh x$ and $\cosh x$ for $x$ (in radians) ranging from 0 to 2 at intervals of 0.0001 .

(1939) XVII + 405 pages; bound in buckram, $\$ 2.00$.

MT4. Tables of Sines and Cosings gor Radian Arguments:

Contains 8 decimal place values of sines and cosines for radian arguments ranging from 0 to 25 at intervals of 0.001 .

(1940) XXIX + 275 pages; bound in buckram, $\$ 2.00$.

MT5. Tables of Sine, Cosine, and Exponential Integrals, Volume I:

Values of these functions to 9 places of decimals from 0 to 2 at intervals of 0.0001 .

(1940) XXVI+444 pages; bound in buckram, $\$ 2.00$.

MT6. Tables of Sine, Cosine, and Exponential Intbgrals, Volume II:

Values of these functions to 9,10 , or 11 significant figures from 0 to 10 at intervals of 0.001 , with auxiliary tables.

(1940) XXXVII + 225 pages; bound in buckram, $\$ 2.00$.

\section{MT7. Table of Natural Logarithms, Volume I:}

Logarithms of the integers from 1 to 50,000 to 16 places.

(1941) XVIII + 501 pages; bound in buckram, $\$ 2.00$.

Payment is required in advance. Make remittance payable to the "National Bureau of Standards", and send with order, using the blank form on the page facing this one for the purpose.

Above prices are for delivery in the United States and its possessions and in countries extending the franking privilege. To other countries the price of MT1 is 65 cents and that of MT2, MT3, MT4, MT5, MT6, and MT7 is $\$ 2.50$ each; remittance to be made payable in United States currency.

Copies of these publications have been sent to various Government depositories throughout the country, such as public libraries in large cities, and colleges and universities, where they may be consulted.

A mailing list is maintained for those who desire to receive announcements regarding new tables as they become available. A list of the tables it is planned to publish will be sent on request. 